\title{
Diálogo Aberto: a experiência finlandesa e suas contribuições
}

\author{
Open Dialogue: the finnish experience and its contributions
}

Luciane Prado Kantorski1, Mario Cardano²

RESUMO O Diálogo Aberto é um método desenvolvido na década de 1980, na Finlândia, para o enfrentamento da crise psicótica. Este artigo é um ensaio teórico que tem como objetivo apresentar o Diálogo Aberto em seus princípios e enquanto prática de saúde mental desinstitucionalizante, enfatizando seu potencial terapêutico, seus resultados e suas contribuições para outros países. Conclui-se que, para a implementação do Diálogo Aberto em outros contextos, é necessária uma mudança organizativa na estruturação dos serviços e uma substantiva mudança cultural no interior da equipe e da comunidade.

PALAVRAS-CHAVE Saúde mental. Transtornos psicóticos. Família.

ABSTRACT The Open Dialogue is a method developed in the 80's, in Finland, to the confrontation of the psychotic crisis. This article is a theoretical essay that aims to present the Open Dialogue in its principles and as a deinstitutionalizing mental health practice, emphasizing its therapeutic potency, results and contributions for other countries. It is concluded that, for the implementation of the Open Dialogue in other contexts, it is necessary an organizational change in the structuring of services and a substantive cultural change within the team and the community.

KEYWORDS Mental health. Psychotic disorders. Family.

1 Universidade Federal de Pelotas (UFPel), Faculdade de Enfermagem - Pelotas (RS), Brasil.

kantorski@pq.cnpq.br

2 Università Degli Studi di Torino, Dipartimento di Culture, Politica e Società Torino, Itália.

mario.cardano@unito.it 


\section{Introdução}

O método do Diálogo Aberto foi desenvolvido por Jaakko Seikkula e sua equipe, no início da década de 1980, na Finlândia, na região oeste da Lapônia, para o enfrentamento da crise psicótica. O Diálogo Aberto engloba uma filosofia e um conjunto de práticas que se diferem radicalmente das convencionais, contribuindo significativamente para o processo de desinstitucionalização naquele país e que podem vir a contribuir em outros contextos (SEIKKULA; ALAKARE; AALTONEN, 2001; SEIKKULA, 2011; SEIKKULA, 2014).

O Diálogo Aberto é baseado em sete princípios. O primeiro consiste na 'ajuda imediata', que pressupõe que haja uma equipe de atenção à crise à disposição, de modo que o primeiro atendimento ocorra nas primeiras 24 horas após o primeiro contato, preferencialmente no domicílio do usuário, com o objetivo de prevenir a hospitalização. $\mathrm{O}$ segundo princípio trata da 'inclusão da rede social do usuário', como a família, os amigos e vínculos significativos em todas as reuniões de tratamento, sem que nenhuma decisão seja tomada fora delas. O terceiro princípio está relacionado com a 'flexibilidade', que deve ser garantida através da adaptação do tratamento às necessidades específicas de cada caso. O quarto princípio é a 'responsabilidade', considerando que o técnico que atende primeiramente o usuário organizará as reuniões sucessivas. O quinto princípio é o da 'tolerância à incerteza', que pressupõe encontros iniciais frequentes (até diários) nos primeiros 10-12 dias de tratamento, como um empenho para evitar decisões precipitadas. Por exemplo, o uso de neurolépticos não é iniciado na primeira reunião. Em vez disso, a sua pertinência deve ser discutida em, pelo menos, três reuniões antes da introdução. $\mathrm{O}$ sexto princípio é o da 'continuidade psicológica', que é garantida em todas as etapas do tratamento. O sétimo princípio é o dialogismo, sendo garantido como foco do tratamento a geração de diálogo entre as pessoas nas reuniões, valorizando a construção conjunta de entendimentos e saídas (SEIKKULA; ALAKARE; AALTONEN, 2001).

O Diálogo Aberto ganhou atenção internacional porque seu uso no tratamento de pessoas com primeiro episódio de psicose é associado, na literatura científica, à redução da incidência do desenvolvimento de sintomas crônicos e de incapacidades decorrentes da psicose e ao uso mínimo de medicação psicotrópica (SEIKKULA ET AL., 2003; SEIKKULA ET AL., 2006; AALTONEN; SEIKKULA; LEHTINEN, 2011; SEIKKULA; ALAKARE; AALTONEN, 2011; THOMAS, 2011). Ainda, são encontrados os estudos de Skalli e Nicole (2011), Gromer (2012) e Lakeman (2014), que são os três estudos de revisão de literatura sobre o Diálogo Aberto, que enfocam, particularmente, os êxitos obtidos no tratamento de pessoas com diagnósticos de crises psicóticas.

Extrapolando a especificidade do mundo acadêmico, os movimentos de usuários e as redes de saúde mental, que, mundialmente, fazem a crítica ao uso abusivo de psicofármacos e apontam seus malefícios, também contribuíram de modo determinante para que os olhares se voltassem para o Diálogo Aberto como alternativa a tal realidade.

Robert Whitaker, em seu livro que se tornou um best seller, 'Anatomy of an epidemic: Magic bullets, psychiatric drugs, and the astonishing rise of mental illness in America', fala dos danos que foram produzidos na América do Norte pelo uso massivo de psicofármacos em resposta a situações de estresse. Whitaker passou um ano e meio pesquisando para a escrita do livro. Ele mantém um site na internet listando alguns estudos relevantes sobre o tema. Essas pesquisas na literatura apoiaram a escrita do livro, que tem um caráter de jornalismo investigativo, tendo sido premiado nessa categoria em 2010. O autor faz uma crítica contundente ao uso inadequado de psicofármacos, evidenciando, além dos danos e perturbações que eles podem causar no cérebro, o aumento de gastos do Estado com pagamento de auxílio por incapacidade produzida pelo uso 
abusivo dessas drogas. Em contraposição, o autor apresenta a experiência finlandesa do Diálogo Aberto como um tratamento eficaz, que reduz o uso de psicofármacos e produz ótimos resultados no enfrentamento das psicoses, questionando os motivos de sua escassa replicação em outros contextos. $\mathrm{O}$ autor conheceu a experiência in loco, pois teve a oportunidade de acompanhar um grupo da Lapônia. Em seu best seller, Whitaker faz uma descrição das bases do método e apresenta alguns resultados das pesquisas do grupo de Seikkula, que comprovam a eficácia do mesmo (WHITAKER, 2010).

Sem dúvida, a repercussão do livro de Robert Whitaker promove uma ampliação da divulgação da experiência do grupo de Seikkula na Finlândia e fortalece o questionamento acerca da necessidade de aprofundamento científico dos conhecimentos sobre as potencialidades e os limites do Diálogo Aberto e de sua replicabilidade em outros contextos.

O contato mais efetivo com o tema do Diálogo Aberto se iniciou no ano de 2015, durante o estágio sênior de um dos autores acompanhando o projeto 'Il dialogo aperto un approccio innovativo nel trattamento delle crisi psichiatriche d'esordio - definizione e valutazione degli strumenti ed organizzativi per la trasferibilità del Dialogo Aperto', coordenado pela Dra. Maria Chiara Rossi e Dr. Giuseppe Salamina do Departamento de Prevenção (ASL TO1 - Azienda Sanitaria Locale di Torino 1), que envolve oito Departamentos de Saúde Mental da Itália (2 em Turim, 1 em Savona, 1 em Trieste, 1 em Modena, 1 na Catania e 2 em Roma). A integração ao projeto de capacitação e pesquisa permitiu a realização de seminários e cursos, inclusive com Jaako Seikkula, professor de psicoterapia do Departamento de Psicologia da University of Jyväskylä, que consistiu em uma aproximação muito rica com o Diálogo Aberto.

Este artigo tem como objetivo apresentar o Diálogo Aberto em seus princípios e enquanto prática de saúde mental desinstitucionalizante, enfatizando seu potencial terapêutico, seus resultados e suas contribuições para outros países.

\section{O Diálogo Aberto e seu potencial terapêutico}

O Diálogo Aberto surge como uma prática de saúde mental em que o cuidado à crise é organizado durante as 24 horas sucessivas ao primeiro contato, e o paciente e sua família são convidados a participar não só do primeiro encontro, mas de todas as reuniões de tratamento. Como mencionado anteriormente, o seu idealizador é Jaakko Seikkula, psicólogo clínico que começou a trabalhar no hospital de Keropudas, em Tornio, em 1981 (SEIKKULA, 2011; SEIKKULA, 2014).

Na Finlândia, a prática psicoterápica vem sendo uma parte da saúde pública importante desde o final dos anos 60, com o trabalho do professor Yrjö Alanen e sua equipe, iniciando com a psicoterapia psicodinâmica individual. A equipe de Turku já enfatizava, no final de 1970, que cada processo de tratamento é único e deveria ser adaptado às diferentes necessidades de cada paciente. $\mathrm{O}$ Diálogo Aberto consiste numa modificação da 'Need-Adapted Treatment of Psychosis', desenvolvida por Alanen (BORCHERS, 2014).

A filosofia do Diálogo Aberto é o desenvolvimento de relações entre as pessoas que estão enfrentando problemas, a família e a rede social envolvida e a oferta de apoio à pessoa em casa, em vez de instituições ou locais de reabilitação. São mais valorizadas as habilidades dos envolvidos de gerar diálogos em reuniões conjuntas, mantendo a comunicação aberta entre as partes, respeitando as vozes de todos os participantes, a paciência de ouvir e de não tomar decisões precipitadas, a capacidade de constantemente enfatizar a situação familiar e as questões da vida cotidiana (SEIKKULA, 2003; BORG ET AL., 2009; ARNKIL; SEIKKULA, 2012).

Nas primeiras iniciativas de reuniões 
abertas na Lapônia, esse espaço era simplesmente um fórum para organizar o tratamento, em vez de baseá-lo em ideias dialógicas. No entanto, a partir do contato com os escritos de Bakhtin, os profissionais perceberam que o autor parecia descrever a mesma experiência em romances de Dostoiévski que aquelas vivenciadas nas reuniões polifônicas com os pacientes, as famílias e a equipe. Havia sempre muitas vozes presentes nas reuniões, e, como evidencia Bakhtin, em uma reunião polifônica, a posição de cada participante, especialmente do autor, muda radicalmente. O Diálogo Aberto reforça que a única maneira de proceder é gerar o diálogo entre todas as vozes dos participantes, e, nessa polifonia, nenhuma voz pode ser mais importante do que a outra (BAKHTIN, 1997).

Conforme afirma Bakhtin (1997), o autor de um romance polifônico não pode controlar a ação dos personagens, e a única maneira de sobreviver é estar em diálogo com eles. Os profissionais tinham um papel importante no tratamento, mas não podiam mais definir os passos, os métodos, as intervenções para suprimir sintomas ou mudar o sistema familiar. Eles passaram a integrar as vozes desse diálogo e essa construção conjunta (SEIKKULA, 2011).

$\mathrm{Na}$ era da medicina baseada em evidências, tudo isso soa muito radical, porque desafia a ideia de que os terapeutas devem escolher o método correto de tratamento e centra-se na ideia de que o diagnóstico emerge do diálogo que ocorre em reuniões conjuntas. $\mathrm{O}$ processo de entendimento, ou seja, chegar a uma compreensão prática de uma forma dialógica por todos os interessados do que o paciente tem, do que aconteceu, por si só, pode ser um processo muito terapêutico (SEIKKULA, 2011).

A abordagem dialógica visa a um processo em que o paciente começa a desempenhar um papel mais importante na determinação de como proceder. Evidencia o risco de negligenciar a fala da pessoa com um transtorno psicótico e não conseguir romper com seu isolamento, reforça que o próprio diálogo precisa criar uma linguagem em que todas as vozes possam ser ouvidas, tanto a do paciente quanto daqueles mais próximos a ele (SEIKKULA, 2002; SEIKKULA; ARNKIL; ERIKSSON, 2003; SEIKKULA, 2008).

Alguns estudos (SEIKKULA; ARNKIL; ERIKSSON, 2003; SEIKKULA, 2008; HOLMESLAND ET AL., 2010; OLSON ET AL., 2012; SEIKKULA; LAITILA; ROBER, 2012; BORCHERS, 2014) ressaltam a importância da polifonia de vozes em abordagens dialógicas e terapias familiares, no sentido de evidenciar a eficácia do dialogismo no tratamento de problemas psicóticos, na depressão, e destacam a mudança do monólogo para o diálogo em situações diversas. Reiteram que, no sistema psicossocial finlandês, essa questão implica a transformação dos papéis profissionais, além do que, a equipe deve trabalhar com todos os casos, incluindo os mais difíceis. Essa nova situação aumenta nos profissionais o interesse por métodos que contribuam para a resolução de situações complexas, incluindo a busca de cooperação ao longo das fronteiras e convidando pessoas da rede pessoal do paciente para participar da resolução de problemas, contribuindo para uma mudança de paradigma.

A partir de uma perspectiva dialógica, a resposta do terapeuta pode vir no sentido de um esforço para alargar a zona de desenvolvimento proximal (SEIKKULA; TRIMBLE, 2005). A ideia de Vygotsky de zona de desenvolvimento proximal consiste no espaço metafórico entre o estudante, que se esforça para aprender novas habilidades um pouco além dos limites de sua capacidade atual, e o professor, que, já tendo dominado as habilidades, oferece-se para apoiar o desenvolvimento das habilidades no aluno. No caso do Diálogo Aberto, a experiência da equipe oferece segurança ao paciente e aos membros da rede para identificar suas habilidades em sustentar a conversa sobre as mais difíceis experiências (SEIKKULA; TRIMBLE, 2005). O fato de os membros da equipe não terem participado dos eventos passados, e que moldaram a atual crise, torna-os menos vulneráveis, 
e isso, somado à experiência acumulada de outras situações, contribui para ofertar segurança ao grupo e gerar diálogo.

$\mathrm{Na}$ fase inicial de tratamento, as decisões são adiadas em favor de expandir a conversa, permitindo ao sistema tolerar a ambiguidade, o que possibilita o surgimento de novas ideias para resolver o problema. O importante no processo são as trocas emocionais. Um ou mais membros da equipe podem conduzir a reunião, que deve ser iniciada com uma questão aberta, partindo de informações que eles podem ter sobre o problema e que devem ser expressas em linguagem simples. O líder, então, oferece uma pergunta aberta a quem gostaria de falar. A forma das perguntas não é pré-planejada; cada próxima pergunta parte da resposta anterior, da repetição da palavra, e se faz lentamente, assegurando o ritmo e o lugar de fala de cada participante. A reunião é concluída com uma síntese do que foi discutido e das decisões tomadas (SEIKKULA; TRIMBLE, 2005).

Para o desenvolvimento da prática dialógica, são elencados doze elementos-chave tomados enquanto critérios de fidelidade para que se efetive o Diálogo Aberto: participação de dois (ou mais) terapeutas na reunião; envolvimento da família e rede social; usar perguntas abertas; responder às coisas ditas pelo paciente; enfatizar o momento; solicitar múltiplos pontos de vista; usar o foco relacional no diálogo; responder aos problemas dialógicos e comportamentais com um estilo concreto e atento aos significados; enfatizar as palavras usadas pelo paciente e sua história, não os sintomas; a conversa entre profissionais (reflexões) deve ocorrer nas reuniões de tratamento; ser transparente; tolerar a incerteza (OLSON; SEIKKULA; ZIEDONIS, 2014).

O nome Diálogo Aberto foi usado pela primeira vez em 1995, para descrever a abordagem à crise através do diálogo, englobando a família inteira e o tratamento centrado na rede social. Isso incluiu dois aspectos: primeiro, as reuniões com paciente, família, rede social e equipe envolvida no problema, que buscavam gerar o novo entendimento através do diálogo. Em segundo lugar, o fornecimento dos princípios básicos para a organização de todo o sistema de tratamento psiquiátrico na Lapônia com base no Diálogo Aberto (SEIKKULA, 2011).

\section{Estudos de avaliação de resultados das práticas de Diálogo Aberto}

Os estudos de resultados da aplicação do Diálogo Aberto em pacientes em primeira crise psicótica e em tratamento com esse método são estudos de follow up, em dois, cinco e dez anos, incluindo grupos de controle (SEIKKULA ET AL., 2003; SEIKKULA ET AL., 2006; AALTONEN; SEIKKULA; LEHTINEN, 2011; SEIKKULA; ALAKARE; AALTONEN, 2011).

Os estudos demonstram que os pacientes que se utilizaram do Diálogo Aberto ficaram hospitalizados por menor número de dias, que os psicofármacos foram utilizados em menor número de casos e que a maioria ( $82 \%)$ dos pacientes não tinha sintomas psicóticos residuais em 2 anos (SEIKKULA ET AL., 2003).

No seguimento de 5 anos, constatou-se que $86 \%$ retomaram os seus estudos ou empregos em tempo integral; $17 \%$ recaíram durante os primeiros 2 anos e 19\% durante os três anos seguintes; e que $29 \%$ fizeram uso de medicação neuroléptica durante alguma fase do tratamento (SEIKKULA ET AL., 2006).

Houve uma diminuição da incidência anual média da esquizofrenia, sendo que o número de novos pacientes esquizofrênicos de longa permanência caiu para zero no contexto estudado (AALTONEN; SEIKKULA; LEHTINEN, 2011).

Durante um período de dez anos, além de surgirem menos pacientes esquizofrênicos, sua idade média foi significativamente menor, e mais de $70 \%$ dos pacientes não tiveram nenhum retorno. Esse resultado permaneceu o mesmo por um período de 
dez anos, envolvendo pacientes diferentes (SEIKKULA; ALAKARE; AALTONEN, 2011).

Os resultados das pesquisas de avaliação da experiência desenvolvida ao longo das últimas décadas na Finlândia comprovam a necessidade de dedicar tempo, trabalho e estudos sobre o Diálogo Aberto. É importante reconhecer que o Diálogo Aberto tem se mostrado potente no enfrentamento do problema de pessoas em crise psicótica, propiciando um manejo mais adequado dos psicofármacos e contribuindo para a inserção social dessas pessoas.

\section{O diálogo aberto e suas contribuições para o mundo}

O Diálogo Aberto, nascido na Finlândia, e os impressionantes resultados obtidos no tratamento da psicose têm despertado o interesse de outros países, como Estados Unidos, Reino Unido, Itália, Polônia, Noruega, Bélgica, Austrália, entre outros.

Retomam-se, a seguir, alguns estudos e relatos de experiências de pesquisadores de diversos países que fizeram um acompanhamento das atividades do Diálogo Aberto na Finlândia e vêm propondo formas de adesão a essa prática, reafirmando a efetividade desse método e a necessidade de sua replicabilidade.

Harlene Anderson, de Houston (Estados Unidos), realizou um acompanhamento da experiência da Lapônia e relatou em seu artigo que, na prática, esse trabalho representa um significativo desafio às crenças e tradições de psicoterapia e abordagens nucleares à psicose. Isso porque ele consiste em uma abordagem dialógica na qual o terapeuta deve respeitar e aprender sobre os pacientes e suas experiências e entendimentos, considerando que isto deve ter precedência sobre a compreensão do terapeuta (ANDERSON, 2002).

Também, num estudo de pesquisadores da Noruega e dos Estados Unidos, são descritos dois modelos de cuidado centrados na pessoa: as práticas de cuidado centradas na pessoa, planejadas nos Estados Unidos, e o modelo finlandês do Diálogo Aberto, destacando seus resultados exitosos por manter o foco no indivíduo (BORG ET AL., 2009).

A partir de uma crítica à falta de acesso das pessoas nos Estados Unidos a tratamentos alternativos à prática padrão de tratamento da psicose, é feita uma revisão narrativa do modelo de tratamento da psicose, adaptado às necessidades do paciente, e do Diálogo Aberto desenvolvido na Finlândia. A análise dos resultados de sete pesquisas mostrou que os resultados do tratamento com o Diálogo Aberto são equivalentes ou superiores àqueles do tratamento padrão. Particularmente, o Diálogo Aberto foi associado a um melhor funcionamento social, a mais emprego, menos dias de hospitalização e menos sintomas para pessoas com primeiro episódio de psicose. Estudos mais recentes apontam para uma associação do Diálogo Aberto com poucos dias de permanência no hospital, o que mostra uma evolução na abordagem e um certo refinamento dessa prática (GROMER, 2012).

Outra pesquisadora da Bélgica fez um seguimento da experiência da Lapônia examinando, também, a natureza do diálogo (OLSON ET AL., 2012). Em um outro estudo autoetnográfico, a pesquisadora descreve as mudanças de pensamento ligadas à sua experiência com o Diálogo Aberto, relacionando essa trajetória à micropolítica dos Estados Unidos na gestão de cuidados de saúde mental; à prática de dialogicidade no Diálogo Aberto; e às mudanças históricas, culturais e científicas que estão incentivando a adaptação do Diálogo Aberto nos Estados Unidos. Menciona, ainda, a participação de Douglas Ziedonis, Jaakko Seikkula e Mary Olson na liderança de um projeto de pesquisa subvencionado sobre Diálogo Aberto do Departamento de Psiquiatria da Escola de Medicina da Universidade de Massachusetts. Evidencia as iniciativas de treinamento de equipes em cuidados comunitários que fazem parte de 
novas iniciativas públicas experimentais, estruturadas prevendo avaliação dos resultados, demonstrando um interesse crescente pelo Diálogo Aberto nos Estados Unidos (OLSON, 2015).

No Sul da Noruega, entre 1998 e 2008, foram implementados três programas para a promoção do Diálogo Aberto na região, e Jaakko Seikkula participou de todos eles, quais sejam: 1) ensinando a base para abrir diálogos; 2) supervisionando clinicamente terapeutas locais e usuários de serviços de saúde mental; 3) concepção de pesquisa e projetos. Segundo a análise desses programas, os dez anos de implementação dessas práticas dialógicas no sul da Noruega produziram resultados positivos relativos à execução, à educação e ao aumento de pesquisas e projetos sobre o tema (ULLAND ET AL., 2014).

No Reino Unido, o interesse pelo Diálogo Aberto vem crescendo continuamente com conferências e experiências pontuais de pequena escala. Um movimento muito significativo começou para intervir nos serviços no sentido de implantar o Diálogo Aberto. Determinadas cidades, como Leeds e Nottingham, possuem grupos de usuários e serviços capacitados no Diálogo Aberto, que estão disponíveis para ensinar e estimular as pessoas a tal prática. Além disso, até sete serviços nacionais de saúde mental da Inglaterra estão estabelecendo equipes-piloto de Diálogo Aberto em cada um dos seus territórios, a fim de participar conjuntamente de um estudo multicêntrico aleatório sobre o tema (RAZZAQUE; WOOD, 2015).

O Diálogo Aberto é apresentado por pesquisadores da Polônia como uma abordagem alternativa que pode contribuir para a redução do uso de neurolépticos e constituir-se como recurso complementar aos tratamentos usuais da esquizofrenia (KŁAPCIŃSKI; WOJTYNSKA; RYMASZEWSKA, 2015).

Em um estudo de revisão sistemática descrevendo diferentes serviços especializados de tratamento de primeiro episódio psicótico e seus resultados em diferentes momentos do acompanhamento, foram identificados cinco programas, todos escandinavos: o projeto Paraquedas, na Suécia; o projeto esquizofrenia Danish National, na Dinamarca; o projeto Opus, também na Dinamarca; projeto Soteria Nacka, na Suécia; e o projeto Diálogo Aberto, na Finlândia. Os programas mostraram resultados encorajadores em termos de diminuição de recaídas, diminuição do tempo de internação e redução do uso de antipsicóticos, principalmente na fase ativa. Porém, um período de tratamento de dois anos não é tempo suficiente para permitir aos pacientes manter as melhorias obtidas durante a fase ativa de um tratamento integrado (SKALLI; NICOLE, 2011).

O Diálogo Aberto despertou o interesse da Austrália, país onde se investiu fortemente em equipes de especialistas para intervenção na psicose, modelo que vem sendo questionado considerando-se as altas taxas de consumo de psicofármacos por pessoas diagnosticadas com psicoses e as altas taxas de mortalidade relacionadas a esse consumo. Um artigo de revisão publicado na Austrália, em 2014, aponta resultados exitosos do tratamento com o Diálogo Aberto para intervenção na crise psiquiátrica com o mínimo de uso de medicação antipsicótica e com melhores respostas, sugerindo sua adoção para realidades que precisam enfrentar tal problemática (LAKEMAN, 2014).

\section{Conclusões}

Na Finlândia, na experiência de mais de trinta anos do oeste da Lapônia, algumas escolhas estratégicas foram adotadas visando a assegurar a continuidade dos cuidados. Foram constituídos serviços de internação com 30 leitos psiquiátricos hospitalares de cuidados agudos e 5 clínicas ambulatoriais onde o Diálogo Aberto é a filosofia de prática, e, em cada unidade, equipes móveis para atendimento à crise estão disponíveis. A maioria dos profissionais foi treinada (por 
cerca de três anos) em terapia familiar sistêmica (BORG ET AL., 2009).

$\mathrm{O}$ questionamento que necessita ser feito consiste nos desafios de colocar em prática em outros países uma abordagem desinstitucionalizante que tem enfrentado com êxito o grave problema de pessoas em crise psicótica no seu domicílio, com o princípio do diálogo e da atenção em rede. Ao mesmo tempo, esse novo método propõe uma mudança significativa, ao fazê-lo de modo que nenhuma decisão seja tomada sem a participação do paciente e sua rede, reduzindo o consumo de psicofármacos e obtendo resultados muito promissores.

Destaca-se que implementar o Diálogo Aberto consiste num desafio que requer

\section{Referências}

\footnotetext{
AALTONEN, J.; SEIKKULA, J.; LEHTINEN, K. The comprehensive open-dialogue approach in western Lapland I: the incidence of non-affective psychosis and prodromal states. Psychosis, Londres, v. 3, n. 3, p. 179191, 2011.
}

ANDERSON, H. In the space between people: Seikkula's open dialogue approach. $J$ Marital Fam Ther, Washington, DC, v. 28, n. 3, p. 279-281, jul. 2002.

ARNKIL, T. E.; SEIKKULA, J. Metodo dialogici nel lavoro di rete: per la psicoterapia di gruppo, il servizio sociale e la didattica. Trento: Erickson, 2012.

BAKHTIN, M. Problemas da poética de Dostoiévski. Rio de Janeiro: Forense Universitária, 1997. uma consistente mudança organizativa em termos de estruturação de serviços, com equipes para atenção à crise disponíveis 24 horas, garantia de continuidade psicológica, tolerância à incerteza, protelamento do uso de neurolépticos, um criterioso processo de capacitação das equipes, entre outras iniciativas. Além disso, torna-se indispensável uma substantiva mudança cultural, que implica rever seriamente as relações de poder entre os profissionais da equipe e destes com pacientes e familiares. Respostas efetivas a problemas complexos, como é o caso de pessoas em crise psicótica, dificilmente podem ser dadas sem que mudanças significativas sejam feitas.
BORCHERS, P. Issues like this have an impact: the need-adapted treatment of psychosis and the psychiatrist's inner dialogue. Jyväskylä: University of Jyväskylä, 2014

BORG, M. et al. Implementing person-centered care in psychiatric rehabilitation: what does this involve? Isr J Psychiatry Relat Sci, Jerusalém, v. 46, n. 2, p. 84-93, 2009.

GROMER, J. Need-adapted and open-dialogue treatments: empirically supported psychosocial interventions for schizophrenia and other psychotic disorders. Ethical Hum Psychol Psychiatry, Nova York, v. 14, n. 3, p. 162-177, 2012. 
HOLMESLAND, A. L. et al. Open dialogues in social networks: professional identity and transdisciplinary collaboration. Int J Integr Care, Londres, v. 10, p. 1-14, 2010.

KŁAPCIŃSKI, M.; WOJTYNSKA, R.; RYMASZEWSKA, J. Open dialogue approach: an alternative to neuroleptics or development of pharmacologically cautious treatment of schizophrenia? J Schizophr Res, Nova Jersey, v. 2, n. 2, p. 1013-1014, 2015.

LAKEMAN, R. The Finnish open dialogue approach to crisis intervention in psychosis: a review. Psychotherapy in Australia, Palm Cove, v. 20, n. 3, p. 26-33, 2014.

OLSON, M. An auto-ethnographic study of 'open dialogue': the illumination of snow. Fam Process, Nova York, v. 54, n. 4 , p. 716-729, 2015.

OLSON, M.; SEIKKULA, J.; ZIEDONIS, D. The key elements of dialogic practice: in open dialogue: fidelity criteria. 2 set. 2014. Disponível em: <http://umassmed. edu/psychiatry/globalinitiatives/opendialogue/>. Acesso em: 19 out. 2016.

OLSON, M. et al. The shift from monologue to dialogue in a couple therapy session: dialogical investigation of change from the therapists' point of view. Fam Process, Nova York, v. 51, n. 3, p. 420-435, 2012.

RAZZAQUE, R.; WOOD, L. open dialogue and its relevance to the NHS: opinions of NHS staff and service users. Community Mental Health, Nova Yok, v. 51, n. 8, p. 931-938, 2015.

SEIKKULA, J. Becoming dialogical: psychotherapy or a way of life? Aust N Z J Fam Ther, Queensland, v. 32, n. 3, p. 179-193, 2011.

Il dialogo aperto: l'approccio finlandese alle gravi crisi psichiatriche. Roma: Giovani Fioriti Editore, 2014.

Inner and outer voices in the present moment of family and network therapy. J Fam Ther, Londres, v. 30, p. $478-491,2008$.
Monologue is the crisis-dialogue becomes the aim of therapy. J Marital Fam Ther, Washington, DC, v. 28, n. 3, p. 283-284, 2002.

Open dialogue integrates individual and systemic approaches in serious psychiatric crises. Smith Coll Stud Soc, [internet], v. 73, n. 2, p. 227-245, 2003.

SEIKKULA, J.; ALAKARE, B.; AALTONEN, J. The comprehensive open-dialogue approach in western Lapland II - long-term stability of acute psychosis outcomes in advanced community care. Psychosis, Londres, v. 3, n. 3, p. 192-204, 2011.

Open dialogue in psychosis I: an introduction and case illustration. J Constr Psychol, Washington, DC. v. 14, n. 4, p. 247-265, 2001.

\section{SEIKKULA, J.; ARNKIL, T. E.; ERIKSSON, E.}

Postmodern society and social networks: open and anticipation dialogues in network meetings. Fam Process, Nova York, v. 42, n. 2, p. 185-203, 2003.

SEIKKULA, J.; LAITILA, A.; ROBER, P. Making sense of multi-actor dialogues in family therapy and network meetings. J Marital Fam Ther, Washington, DC, v. 38, n. 4, p. 667-687, 2012.

SEIKKULA, J.; TRIMBLE, D. Healing elements of therapeutic conversation: dialogue as an embodiment of love. Fam Process, Nova York, v. 44, n. 4, p. 461-475, 2005.

SEIKKULA, J. et al. Five-year experience of first-episode non affective psychosis in open-dialogue approach: treatment principles, follow-up outcomes, and two case studies. Psychother Res, Nova York, v. 16, n. 2, p. 214-228, 2006.

SEIKKULA, J. et al. Open dialogue approach: treatment principles and preliminary results of a two - year follow-up on first episode schizophrenia. Ethical Hum Sci Serv, Nova York, v. 5, n. 3, p. 163-182, 2003.

SKALLI, L.; NICOLE, L. Specialized first-episode psychosis services: a systematic review of the literature. Encephale, Paris, v. 37, supl. 1, p. 66-76, 2011. 
THOMAS, S. P. Open-dialogue therapy: can a Finnish approach work elsewhere? Issues Ment Health Nurs., Nova York, v. 32, n. 10, p. 613, 2011

ULLAND, D. et al. Generating dialogical practices in mental health: experiences from southern Norway, 1998-2008. Adm Policy Ment Health, Nova York, v. 41, p. 410-419, 2014
WHITAKER, R. Anatomy of an epidemic: magic bullets, psychiatric drugs, and the astonishing rise of mental illness in America. Nova York: Crown Publishing Group, 2010.

Recebido para publicação em outubro de 2016 Versão final em março de 2017

Conflito de interesses: inexistente

Suporte financeiro: Projeto financiado pelo Conselho Nacional de Desenvolvimento Científico e Tecnológico (CNPq) - processo no 6458/2014-09 